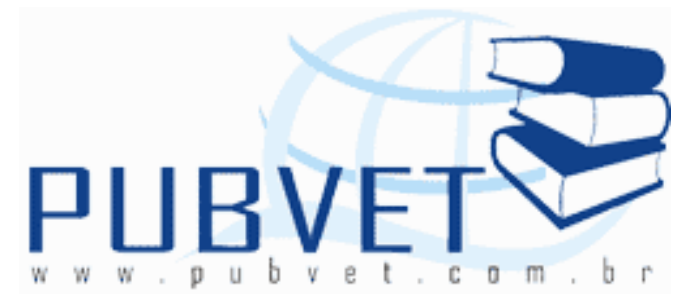

PUBVET, Publicações em Medicina Veterinária e Zootecnia.

\title{
Tratamento de traumatismo craniano em gambá Didelphis albiventris Linnaeus, 1758 (Marsupialia, Didelphidae)
}
André Luiz Quagliatto Santos ${ }^{1}$, Juliana Macedo Magnino Silva ${ }^{2}$, Heloísa Castro Pereira $^{3}$, Liliane Rangel Nascimento ${ }^{2}$, Lorena Tannús Menezes ${ }^{2}$, Árthur Paulino Sanzo Kaminishi

Laboratório de Ensino e Pesquisa em Animais Silvestres - LAPAS, FAMEV/UFU, email: quagliatto@famev.ufu.br 1. Docente. 2. Mestrandos. 3. Médica veterinária residente.

\section{Resumo}

Traumatismo craniano consiste em lesões agudas e crônicas ao encéfalo, incluindo os hemisférios cerebrais, cerebelo e tronco cerebral. É relatado um caso de um gambá macho Didelphis albiventris, apresentando movimentos descoordenados e pupila dilatada hiperestesia no abdômen, falta de coordenação e paralisia nos membros pelvinos. O tratamento prescrito foi Dexametasona e Ácido tranexâmico nas doses de $1 \mathrm{mg} / \mathrm{Kg}$ e $10 \mathrm{mg} / \mathrm{Kg}$ respectivamente. Observou-se efetividade do tratamento do gambá vítima de traumatismo craniano.

Palavras-chave: marsupial, traumatologia, dexametasona, ácido tranexâmico. 

2011.

\title{
Treatment of head injury in opossum Didelphis albiventris Linnaeus, 1758 (Marsupialia, Didelphidae)
}

\begin{abstract}
Head trauma is a acute and chronic injuries in the brain, including cerebral hemispheres, cerebellum and brainstem. Were report a case of a male opossum Didelphis albiventris, showing uncoordinated movements, dilated pupils, soreness in the abdomen, lack of coordination and paralysis of pelvic limbs. The treatment was prescribed tranexamic acid and dexamethasone in doses of $1 \mathrm{mg} / \mathrm{kg}$ and 10 $\mathrm{mg} / \mathrm{kg}$ respectively. Were observed effectiveness of the treatment of the opossum victim of head trauma.
\end{abstract}

Keywords: marsupial, traumatology, dexamethasone, tranexamic acid.

\section{INTRODUÇÃO}

O gambá de orelha preta ou comum, Didelphis albiventris, é um marsupial de hábitos solitários e noturnos, refugiando-se de dia nos ocos de árvores, entre raízes, sob montes de folhas secas ou em forros de casas. São terrestres e utilizam o habitat arbóreo ocasionalmente, para procura de alimentos (CABRERA E YEPES, 1960; HUNSAKER, 1977; CHEREM et al., 1966)

Traumatismo craniano consiste em lesões agudas e crônicas ao encéfalo, incluindo os hemisférios cerebrais, cerebelo e tronco cerebral. As manifestações clínicas dependem da natureza da lesão. O trauma difuso ao encéfalo é frequentemente associado com lesão axonal difusa ou coma pós-traumático. As lesões localizadas podem estar associadas com manifestações neurocomportamentais; hemiparesia ou outras deficiências neurológicas focais. Os sinais e sintomas de uma lesão na cabeça podem ocorrer imediatamente ou se desenvolver lentamente no decorrer de várias horas (BOUMA et al., 1991). 
SANTOS, A.L.Q. et al. Tratamento de traumatismo craniano em gambá Didelphis albiventris Linnaeus, 1758 (Marsupialia, Didelphidae). PUBVET, Londrina, V. 5, N. 17, Ed. 164, Art. 1110, 2011.

Todo trauma craniano deve ter acompanhamento médico durante um prolongado período de tempo (BOUMA et al., 1991). Esse acompanhamento deve iniciar com um detalhado exame físico neurológico e radiográfico. A observação minuciosa do animal deve ser estabelecida.

Caso tenha se instalado o "foco epilético", drogas anticonvulsivantes devem ser administradas sob monitoração do clínico veterinário (OSWALD e GUIMARAES, 2001; FRASER, 2001). Observe-se que estas drogas têm controle pelo Ministério da Saúde necessitando, portanto receituário médico especial.

Uma das seqüelas do traumatismo craniano é a convulsão, podendo ocorrer também paresias e paralisias entre outros sintomas. Animais com tratamento adequado e monitoramento de rotina podem ter vida normal (BOUMA et al., 1991). Nesse relato foram descritos os sinais clínicos observados e o devido tratamento num caso de traumatismo craniano em gambá.

\section{RELATO DO CASO}

Foi atendido no Ambulatório de Animais Silvestres do Hospital Veterinário da Universidade Federal de Uberlândia um gambá macho, adulto de $1,5 \mathrm{Kg}$ encontrado em uma residência em decúbito lateral, executando movimentos descoordenados e pupila dilatada. O animal apresentou hiperestesia no abdômen, falta de coordenação e paralisia nos membros pelvinos, não havendo sensibilidade nesta região. Foi observado a presença de sangue na região ocular, ataxia e nistagmo vertical além da presença de sangue nos ouvidos. O animal foi mantido em repouso e alimentado uma vez ao dia com ovo cru e camungongos triturados. Os parâmetros vitais foram monitorados em intervalos de uma hora. 0 tratamento prescrito foi Dexametasona e Ácido Tranexâmico nas doses de $1 \mathrm{mg} /$ $\mathrm{Kg}$ e $10 \mathrm{mg} / \mathrm{Kg}$ respectivamente, ambos administrados pela via intramuscular, uma vez ao dia, durante 3 dias. 
SANTOS, A.L.Q. et al. Tratamento de traumatismo craniano em gambá Didelphis albiventris Linnaeus, 1758 (Marsupialia, Didelphidae). PUBVET, Londrina, V. 5, N. 17, Ed. 164, Art. 1110, 2011.

\section{RESULTADOS E DISCUSSÃO}

O acúmulo de forças de impacto geradas pelo traumatismo craniano na base do crânio é responsável pela formação de contusões no tronco encefálico (BOUMA et al., 1991). A história, o exame físico e a investigação imagenológica do paciente proporcionam evidências adicionais para alguns dos mecanismos propostos para explicar a oftalmoplegia bem como outros sintomas associados à região ocular (BONILHA et al., 2002).

Para BOUMA et al. (2002) o manuseio terapêutico tem embasamento no conhecimento das injúrias cerebrais primárias e secundárias. As injúrias primárias representam os efeitos imediatos e/ou irreversíveis da dissipação de energia dentro da substância cerebral. A vasta maioria desses fenômenos ocorre dentro dos millisegundos do impacto ou penetração. As injúrias primárias incluem as disrupturas neuronais ou gliais diretas, as injúrias vasculares e as injúrias de partilhação (explosão temporal, lacerações). As injúrias primárias são melhores compreendidas quando separadas em focais (hematoma, contusão, laceração, hemorragia), ou difusas (hemorragias petequiais múltiplas, inchaço cerebral, disfunção transitória (confusão, amnésia, inconsciência) e lesão axonal persistente (inconsciência longa, lesão axonal difusa). Existe uma forte evidência de que as lesões primárias podem ocorrer em tempo posterior ao trauma, tal como horas, conhecidas como lesões primárias tardias.

Imediatamente depois do insulto primário, uma série de eventos reativos inicia, alguns causam aceleração ou aumento da injúria celular inicial e outros causam novas injúrias. Esta cascata complexa de eventos constitui as injúrias cerebrais secundárias, que podem ser categorizadas de várias formas. Além disso, alterações metabólicas, desequilíbrios fluidos ou eletrolíticos, e infecção local ou sistêmica podem agravar a injúria traumática. No nível celular, ocorrem reações bioquímicas que podem resultar em morte das células parcialmente 
SANTOS, A.L.Q. et al. Tratamento de traumatismo craniano em gambá Didelphis albiventris Linnaeus, 1758 (Marsupialia, Didelphidae). PUBVET, Londrina, V. 5, N. 17, Ed. 164, Art. 1110, 2011.

injuriadas. Injúria cerebral severa está associada com um insulto metabólico sistêmico (BOUMA et al., 1991).

É importante estar atento às necessidades calóricas do animal dando a ele suporte nutricional.

Os corticosteróides exercem efeitos sobre quase todas as células, influenciando o metabolismo protéico, lipídico e glicídico, o balanço hidroelectrolítico, as funções cardiovascular, renal, da musculatura esquelética, do sistema nervoso e de praticamente todos os tecidos e órgãos. Desempenham um papel importante na homeostasia dos estímulos nóxicos internos e externos.

Oswald e Guimarães, 2001 e Fraser, 2001 a dexametazona é indicada na profilaxia das convulsões, já que a presença destas produz hipóxia e edema cerebral, definindo piora substancial das condições do paciente. Também a agitação psicomotora deve ser tratada com sedativos, porque determina efeitos semelhantes (hipóxia e edema cerebral) embora por mecanismos diferentes.

Horrow et al. (1990) o ácido tranexâmico é usado no controle e prevenção de hemorragias ligadas a várias áreas. Estudos comprovam que doses de ácido tranexâmico são eficazes na profilaxia de sangramentos.

No caso descrito, observou-se efetividade do tratamento do gambá vítima de traumatismo craniano.

\section{CONCLUSÃO}

O traumatismo craniano, mesmo se enquadrando em um prognóstico desfavorável pôde ser revertido através de monitoramento e tratamento adequado acompanhados por médicos veterinários no caso descrito em Didelphis albiventris. 


\section{REFERÊNCIAS}

BONILHA, L.; FERNANDES, Y. B.; MATTOS, J. P.; BORGES, W.A.; BORGES, G. Bilateral internuclear ophthalmoplegia and clivus fracture following head injury - Case report Arquivo Neuropsiquiatric, v. 60, n. 1, p.636-638, Out. 2002.

BOUMA, G.J.; MUIZELLAR, P.; CHOI, S.C; NEWLON, P.G.; YOUNG, H.F. Cerebral circulation and metabolism after severe traumatic brain injury: the elusive role of ischemia. Journal of Neurosurgery, v.75, n.5, p. 685-693, Fev. 1991.

CABRERA, A. YEPES, J. Mamíferos Sud Americanos. Buenos Aires: Departamento Público Cientista Argentino, 1960, 187p. v.1

CHEREM, J. J.; GRAIPEL, M. E.; MENEZES, M. E.; SOLDATELI, M. Observações sobre a biologia do gambá (Didelphis albiventris) na Ilha de Ratones Grande, Estado de Santa Catarina, Brasil.

Biotemas, Porto Alegre, v.9, n. 2, p. 47-56, Jan. 1996.

HUNSAKER II, D. Ecology of new world marsupials. In: HUNSAKER II, D. (eds.) The Biology of Marsupials. New York: Academic. Press, 1977, p. 95-156.

OSWALD, W.; GUIMARÃES, S. Terapêutica medicamentosa e suas bases farmacológicas, $4^{a}$ ed.Lisboa: Porto Editora,v. 49, 2001, p. 716-718.

FRASER, C. Manual Merck de Veterinária, Rocca, 2001.

HORROW, J.C.; HLAVACEK, J.; STRONG, M.D.; BRODSKI, I.; GOLDMAN, S. M.;GOEL, I.P. Prophylatic tranexamic acid decreases bleeding after cardiac operations. Journal of Thoracic and Cardiovascular Surgery. v. 99, p. 70-74, Fev. 1990. 